\title{
EFTEM Analysis of FIB Sections: an Important Tool in Nanotechnology
}

\author{
J. Mayer**** and T.E. Weirich* \\ * Central Facility for Electron Microscopy, RWTH Aachen University, Ahornstr. 55, \\ 52074 Aachen, Germany \\ **Ernst Ruska-Centre for Microscopy and Spectroscopy with Electrons, Research Centre Juelich, \\ 52425 Juelich, Germany
}

\begin{abstract}
Although recent instrumental developments have clearly advanced the ultimate energy and spatial resolution limits in spectroscopic studies in the STEM mode much beyond the corresponding limits in EFTEM, there are still many different applications in which EFTEM is the method of choice owing to the fast two-dimensional data acquisition and analysis (see e.g. [1]). EFTEM offers substantial advantages for the analysis of the elemental distribution in complex two- or threedimensional nanostructures, in the latter case even in combination with tomography. Moreover, series of images obtained by electron spectroscopic imaging (ESI) can be analyzed quantitatively to reveal information on the chemical composition or even the bonding state of the different elements [2,3]. Though the energy resolution in ESI images is limited to about $1 \mathrm{eV}$ and the spatial resolution to about $0.5 \mathrm{~nm}$, there is still a large number of applications in which this is absolutely sufficient and the advantages of fast 2 -d data acquisition prevail.
\end{abstract}

An extremely useful synergy has emerged from the use of FIB workstations for the specimen preparation in EFTEM studies. Well-prepared FIB sections have an ideal thickness for EFTEM studies, and whereas the FIB specific damage interferes with the capabilities of many high resolution TEM techniques, the chemical composition is usually not modified to an extent which exceeds the resolution limits of EFTEM discussed above. In particular in nanotechnology, where it is frequently necessary to prepare cross sections from an area which has to be localized with a precision of about ten nanometres, the combination of FIB and EFTEM has proven to be extremely useful. As an example, Fig. 1 shows an elemental distribution image from one of the smallest transistors ever manufactured in Europe [4]. The transistor possesses an electrically variable shallow junction MOSFET design and the characteristic size parameter is the gate width measured at the bottom of the lower gate electrode (arrowed). Our investigations showed that this can best be measured as the chemical width of the polysilicon gate between the $\mathrm{SiO}_{2}$ barrier layers and a value of $12 \mathrm{~nm}$ was obtained. As a second example, Fig. 2a shows the schematical design and Fig. 2b an experimental cross section through an MRAM nonvolatile memory cell based on ferromagnetic Co electrodes separated by a $2 \mathrm{~nm}$ thick $\mathrm{Al}_{2} \mathrm{O}_{3}$ tunneling barrier [5]. As an example for a quantitative analysis of ESI image series, Fig. 3 shows a spectrum line profile across an ohmic Al-Ti contact on $\mathrm{GaN}$ [6]. The analysis revealed that nitrogen-interdiffusion in the Ti interfacial layer with a thickness of only $3 \mathrm{~nm}$ is responsible for the ohmic behavior of the contact.

\section{References}

[1] P. Kohler-Redlich and J. Mayer, in: High-resolution imaging and spectrometry of materials, F. Ernst, M. R, hle (eds.), Springer Series in Materials Science, Springer, Berlin (2002), 119-188.

[2] J. Mayer and J.M. Plitzko, J. Microscopy 183, 2 - 8 (1996)

[3] J. M. Plitzko and J. Mayer, Ultramicroscopy $\underline{78}$, 207-219 (1999).

[4] Sample courtesy of W. Henschel, H. Kurz, Advanced Microelectronic Centre Aachen (AMICA). 
[5] Sample courtesy of U. R diger, G. G, ntherodt, Inst. Physics, RWTH Aachen University. [6] M. Pidun et al., Appl. Surf. Sci. 179, 213-221 (2001).
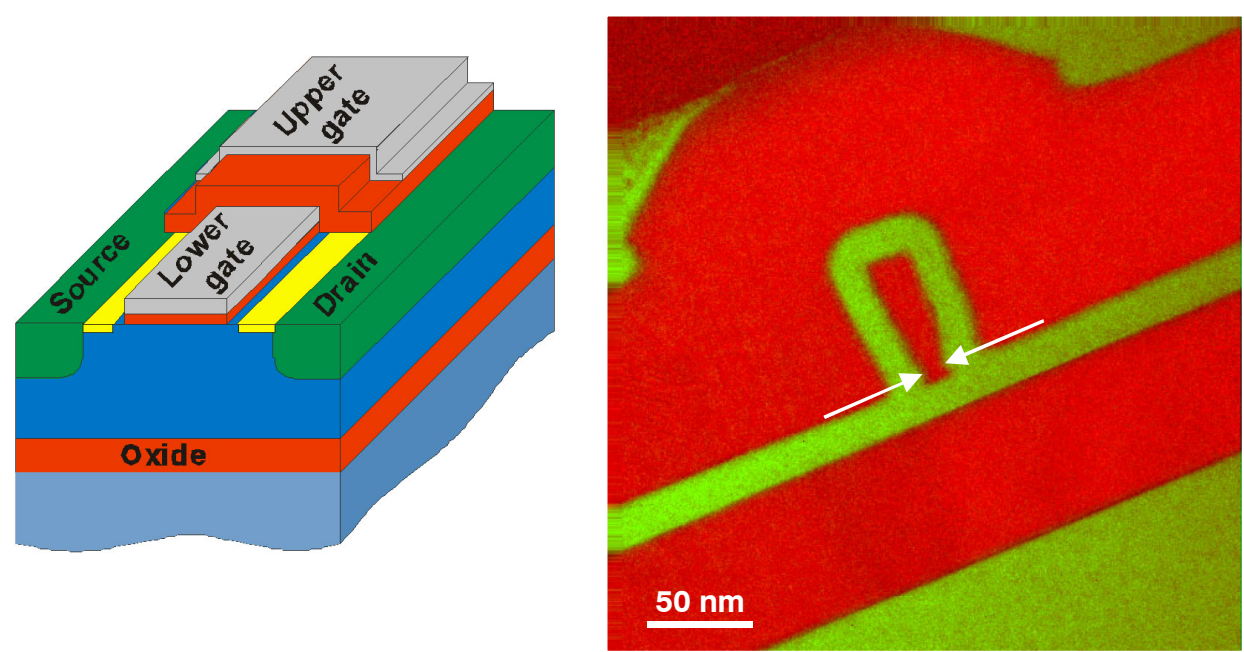

Fig. 1. Schematic design and elemental distribution image of an electrically variable shallow junction MOSFET transistor ( $\mathrm{Si}$ : red, O: green). The gate width (arrowed) was measured as the chemical width between the $\mathrm{SiO}_{2}$ barriers and a value of $12 \mathrm{~nm}$ was obtained [4].

Fig. 2. Schematic design and elemental distribution image of a cross-section through a MRAM memory cell (Si: blue, Co: red, O: green). The two ferromagnetic Co-layers and the continuous $\mathrm{Al}_{2} \mathrm{O}_{3}$ tunnel barrier are clearly visible.

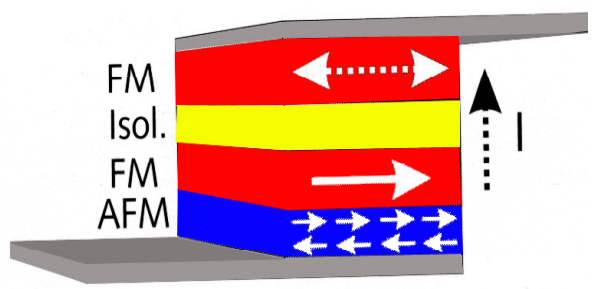

Interconnect Line

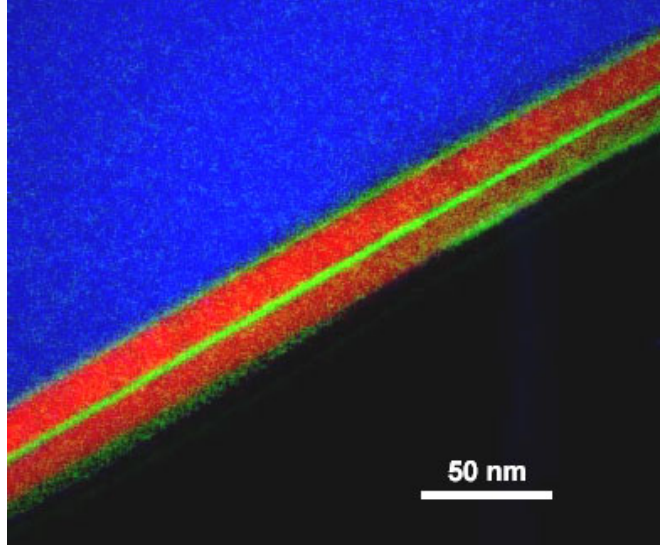

Fig. 3. Spectrum line profile across an ohmic Al-(Ti)-GaN contact structure. The Ti-L edge of the $\mathrm{Ti}$ layer with a thickness of $3 \mathrm{~nm}$ is visible at the interface and quantitative evidence of $\mathrm{N}$-interdiffusion from the $\mathrm{GaN}$ substrate was obtained. The O-K edge emerges from partial oxidation of the Al-layer [6]. 